\title{
Conflicting Effects of Climate and Vector Behavior on the Spread of a Plant Pathogen
}

Matthew P. Daugherty, Department of Entomology, University of California, Riverside, CA 92521; and Adam R. Zeilinger and Rodrigo P. P. Almeida, Department of Environmental Science, Policy and Management, University of California, Berkeley, CA 94720

Accepted for publication 31 March 2017.

\section{ABSTRACT}

Local climatic conditions are important determinants of disease dynamics through effects on vector population performance or distribution. Yet, climate may also be epidemiologically significant due to effects on host-pathogen infection dynamics. We developed a model to explore interactive effects between climatemediated acceleration in disease phenology (i.e., faster incubation or symptom onset) and vector preference based on host symptom status. Higher incubation rates favored pathogen outbreaks, but more rapid symptom onset may constrain spread if vectors avoid symptomatic hosts. Next, we tested whether warmer conditions favored greater spread of the plant pathogen, Xylella fastidiosa, by its leafhopper vector, Graphocephala atropunctata. Inoculated and healthy plants were reared in two temperature-controlled greenhouses. At six times postinoculation, a healthy and inoculated plant were exposed to noninfective vectors, after which pathogen spread was evaluated. Incubation rate and symptom onset in infected hosts was significantly accelerated at higher temperature. Although there was a tendency for greater pathogen spread at higher temperature, the effect depended on time since inoculation. In later introductions, after disease symptoms manifest, vectors were more likely to be found on healthy hosts. Vector avoidance of symptoms, particularly for hosts reared at higher temperature, constrained pathogen spread at later introductions. These results indicate that climate and vector behavior may mediate interactively pathogen spread. Further consideration of such epidemiological complexities is needed to predict adequately the consequences of climate change for disease dynamics.
Evidence linking climate to vector-pathogen-host interactions (Lopes et al. 2009; Richards et al. 2007; Savage et al. 2011) has led to the general perception that climate change will alter the distribution and dynamics of infectious diseases. Yet, notable gaps remain in our understanding of the precise manner in which pathosystems will respond to a changing climate, undermining our ability to generate specific predictions (Lafferty 2009; Rohr et al. 2011).

The literature is replete with examples of links between climate and transmission efficiency (i.e., vector competence), particularly for the inoculation phase of transmission (Daugherty et al. 2009; Richards et al. 2007), which is often positively related to temperature because of increased vector feeding rate or biting frequency (Son et al. 2010) or increased extrinsic incubation rate (i.e., reduced time to vector infectiveness) (Carpenter et al. 2011). The

Corresponding author: M. P. Daugherty; E-mail address: matt.daugherty@ucr.edu

*The $e$-Xtra logo stands for "electronic extra" and indicates that one supplementary appendix, one supplementary figure, and three supplementary files are published online.

(C) 2017 The American Phytopathological Society efficiency with which vectors acquire a pathogen from infected hosts might also covary with temperature due to differences in vector feeding activity or, indirectly, by affecting the intrinsic incubation rate (i.e., host incubation rate). Climate is well known to influence transmission dynamics (Canto et al. 2009) due to pathogen multiplication rates (Feil and Purcell 2001; Munyaneza et al. 2012) and infection persistence (Lieth et al. 2012; Lopes et al. 2009) that depend on temperature. Yet, studies are lacking that evaluate explicitly whether such effects of climate alter disease dynamics. Moreover, other ecological contingencies exist that may mediate the influence of climate on host-pathogen dynamics and spread.

One such contingency for vector-borne pathogens concerns the potential for climatic conditions to influence simultaneously disease onset and vector behavior. Climate can affect the pace of symptom onset (i.e., host latent period) and disease symptom severity in infected hosts (Lovell et al. 2004). Effects on host symptomology may be important because vectors often exhibit orientation or feeding preferences for individual hosts based on host infection status. Such preferences are well documented for arthropod vectors of plant pathogens (Blua and Perring 1992; Ingwell et al. 2012; Mauck et al. 2010), with visual cues (Daugherty et al. 2011) or other host phenotypic changes (i.e., chemical volatiles) (Eigenbrode et al. 
2002) determining the attractiveness of infected hosts. Preference for infected hosts tends to favor disease outbreaks, whereas avoidance of infected hosts should constrain outbreaks (McElhany et al. 1995; Sisterson 2008). Yet host phenotype following infection need not be consistent over time (Blua and Perring 1992), leading to concomitant changes in the magnitude or even trajectory (i.e., switches between preference and avoidance) of vector preference as disease progresses (Werner et al. 2009). Thus, if host incubation period and symptom onset depend on local environmental conditions, climate may interact with vector behavior to determine disease incidence. We evaluated this hypothesis using a combination of epidemiological modeling and an empirical test with a generalist plant pathogen and its leafhopper vector.

Xylella fastidiosa is a xylem-limited bacterium that is pathogenic to a wide variety of plants, including grapevines, in which it causes Pierce's disease (Purcell 1997). Multiplication of the bacterium plugs xylem vessels, which leads to leaf scorch symptoms, shoot dieback, and plant death (Purcell 1997). In parts of California the most dominant vector is the blue-green sharpshooter, Graphocephala atropunctata (Purcell 1975). This native xylem-sap feeding insect is more efficient at transmitting $X$. fastidiosa to grapevines than are other vectors, including the invasive glassy-winged sharpshooter (Homalodisca vitripennis) (Daugherty and Almeida 2009).

Multiple lines of evidence suggest that climate, particularly temperature, plays an important role in X. fastidiosa epidemiology. There are direct effects on vector performance (Son et al. 2009), density (Gruber and Daugherty 2013), feeding rate (Son et al. 2010), and transmission, which is generally more efficient at higher temperatures (Daugherty et al. 2009). In addition, temperature governs $X$. fastidiosa host infection dynamics. A greater proportion of grapevines recover from $X$. fastidiosa infection under colder overwintering conditions (Lieth et al. 2012), whereas $X$. fastidiosa multiplication rates are generally higher under warmer conditions (Feil and Purcell 2001). This last result is notable because $G$. atropunctata acquisition efficiency depends on host infection level, with a threshold of approximately $10^{4} \mathrm{CFU} / \mathrm{g}$ of plant tissue required for efficient acquisition (Hill and Purcell 1997). Collectively, these results suggest that warmer conditions may exacerbate Pierce's disease incidence.

G. atropunctata host preference may also be epidemiologically significant. Sharpshooters exhibit preferences for host species and grapevine variety (Purcell 1981) and strong within-host feeding-site preferences that may underlie acquisition efficiency (Daugherty et al. 2010). In addition, research has documented sharpshooter preferences based on host disease symptom status. Sharpshooters avoid symptomatic hosts (Marucci et al. 2005), but not asymptomatic infected hosts, using visual cues associated with leaf scorch symptoms (Daugherty et al. 2011). Avoidance of symptomatic hosts may constrain pathogen acquisition and, therefore, temper disease incidence compared with what it would be in the absence of symptoms. However, to date no studies have measured pathogen transmission and spread over a gradient of disease symptoms.

Despite ample evidence that climate affects host-pathogen infection dynamics and host symptomology, and further evidence of vector response to host symptomology, the net epidemiological effect is not well understood. Therefore, we used a combination of modeling and experiments to clarify potential interactions between climate and vector behavior with respect to disease dynamics. First, we modeled the effects of host incubation rate, symptom onset, and vector preference to understand the dynamic consequences of climate for disease incidence. Next, to test the qualitative predictions from the model, we conducted an experiment with $G$. atropunctata and $X$. fastidiosa to estimate pathogen spread over a temperature-associated gradient in disease.

\section{MATERIALS AND METHODS}

Epidemiological modeling. To better understand the individual and combined effects of climate and vector behavior on disease dynamics, we used a variation on a vector-borne epidemiological model (Zeilinger and Daugherty 2014):

$$
\begin{aligned}
& \frac{d S}{d t}=\mu(E+C+I)-\frac{\beta S V}{(p I+S+E+C)} \\
& \frac{d E}{d t}=\frac{\beta S V}{(p I+S+E+C)}-(\gamma+\mu) E \\
& \frac{d C}{d t}=\gamma E-(\delta+\mu) C \\
& \frac{d I}{d t}=\delta C-\mu I \\
& \frac{d U}{d t}=\nu V-\frac{\alpha p I U}{(p I+S+E+C)}-\frac{\alpha C U}{(p I+S+E+C)} \\
& \frac{d V}{d t}=\frac{\alpha p I U}{(p I+S+E+C)}+\frac{\alpha C U}{(p I+S+E+C)}-v V
\end{aligned}
$$

where $S$ denotes uninfected "healthy" or "susceptible" hosts, $E$ denotes latently "exposed" hosts that have been infected with the pathogen but are neither infectious nor showing symptoms, $C$ denotes "asymptomatic-infectious" hosts that are an acquisition source but are not yet showing symptoms, $I$ denotes "symptomaticinfectious" hosts that are both infectious and diseased, $U$ denotes "noninfective" vectors (i.e., those that have not acquired the pathogen), and $V$ denotes "infective" vectors. The parameters $\mu$ and $v$ denote host and vector turnover rates (i.e., loss of infection or mortality), respectively, and $\alpha$ and $\beta$ correspond with acquisition and inoculation rates, respectively. Acquisition was assumed to be similar for asymptomatic- and symptomatic-infectious hosts, which may not always be true (Zeilinger and Daugherty 2014). The parameter $p$ describes vector preference based on host infection phenotype. When $p=1$, vector contact with symptomatic hosts (i.e., $I$ ) versus any of the asymptomatic host categories (i.e., $S, E$, or $C$ ) is proportional to the relative density of the two phenotypes, when $p>1$ vectors are disproportionately more likely to contact symptomatic hosts, and when $p<1$ vectors are disproportionately less likely to contact symptomatic hosts. We assumed that vector preference does not differ based on vector infectivity (but see Ingwell et al. 2012) nor that vector preference is affected directly by warming but rather is indirectly affected by changes in host plant quality (e.g., disease symptoms). Finally, $\gamma$ describes the rate at which exposed hosts develop to become infectious (i.e., asymptomatic incubation rate), and $\delta$ is the rate at which asymptomaticinfectious hosts become symptomatic (i.e., rate of symptom onset).

We investigated the epidemiological consequences of climate over gradients in the rates at which exposed and asymptomatic-infectious hosts transitioned to asymptomatic-infectious and symptomaticinfectious states, respectively. In other words, $\gamma$ (incubation rate) and/or $\delta$ (rate of symptom onset) were assumed to be positively related to temperature, as appears to be the general case for $X$. fastidiosa incubation and Pierce's disease symptom onset in grapevines (Feil and Purcell 2001; Lieth et al. 2012).

Model behavior was evaluated primarily relative to whether an outbreak was favored and the initial outbreak dynamics, rather than 
investigating effects on equilibrium prevalence, to more directly match up with the experiment conducted in the second part of the study. Specifically, transient dynamics were evaluated using numerical simulations throughout much of parameter space, especially for $\gamma, \delta$, and $p$, but with generally low initial infection prevalence in the vector and host (i.e., $E_{0}, C_{0}, I_{0}$, and $V_{0}$ near zero). In addition, we explored effects on the pathogen reproduction number, $R_{0}$, as a convenient metric of the relative potential for disease outbreak to occur (Supplementary Appendix):

$$
R_{0}=\frac{\alpha \beta \gamma T(\mu+\delta p)}{\sqrt{\alpha \beta \gamma \mu \nu N T(\gamma+\mu)(\delta+\mu)(\mu+\delta p)}}
$$

where $N$ is the total density of hosts, and $T$ is the total density of vectors, both of which were assumed to be constants. In a deterministic setting, as is the case here, disease outbreak occurs if $R_{0}$ is greater than 1 .

Experiment with $G$. atropunctata and $X$. fastidiosa. To evaluate the qualitative predictions of the model, we tested the effect of incubation temperature on the likelihood of pathogen spread over time in the $X$. fastidiosa-grapevine-sharpshooter system. Early in the spring we propagated approximately 200 own-rooted grapevine cuttings ('Cabernet Franc') in 10-cm-square pots filled with Supersoil potting soil (Rod Mclellan Company, San Mateo, CA). After 1 month of growth, we mechanically inoculated half of the plants with two 10- $\mu$ l droplets of a turbid suspension of $X$. fastidiosa (STL isolate, originally isolated from a symptomatic grapevine in Napa Valley, CA) and SCP buffer. The other half of the plants were inoculated only with SCP buffer. One week later, half of the pathogen-inoculated and half of the healthy plants were moved into two greenhouses at UC Berkeley's Jane Gray Research Greenhouse (Berkeley, CA) set to different temperatures on a 16:8 h (day/night) photoperiod. In a high temperature room, day temperature was set to $32^{\circ} \mathrm{C}$ and night was $21^{\circ} \mathrm{C}$. Corresponding conditions in a low temperature room were set to $21^{\circ} \mathrm{C}$ day and night. Plants remained in these rooms until use in transmission trials, and we regularly repositioned the plants within the rooms to ensure they all were exposed to similar average conditions.

In order to estimate treatment effects on incubation rate, symptom onset, and pathogen spread, we evaluated inoculated plants and conducted transmission trials at six successive dates, approximately $3,4,8,10,14$, and 16 weeks postinoculation. The earliest dates were believed to be less than the time required for symptoms to develop (i.e., less than the latent period) and for high pathogen populations to develop (i.e., less than the incubation period) in grapevines raised in the greenhouse (Hill and Purcell 1995). Conversely, the later dates should allow sufficient time for strong symptoms and high infectiousness to manifest in the inoculated vines. Trials were conducted in a common intermediate temperature room (day $=27^{\circ} \mathrm{C}$, night $=21^{\circ} \mathrm{C}$ ) to ensure that pathogen spread was not confounded by direct effects of temperature on sharpshooter behavior and transmission (Daugherty et al. 2009; Son et al. 2010).

At each of the six dates, one $X$. fastidiosa-inoculated plant and one healthy plant from a given temperature treatment were placed into a $60 \times 60 \times 60 \mathrm{~cm}$ mesh and clear plastic cage (Bugdorm 2, Megaview Sciences Inc., Taiwan). In establishing these pairs, care was taken to choose plants of similar size. Afterward, five G. atropunctata adults were introduced into each cage, placed in the center of the cage on the floor. Sharpshooters were from a colony established from insects collected earlier that summer along the Russian River near Forestville, CA, and then raised on healthy sweet basil plants (Ocimum basilicum L.). Previous tests indicated that the vectors were free from $X$. fastidiosa infection. Vector groups remained in the cages for 7 days.
At the end of each trial we counted the number of vectors on the inoculated and healthy plants, and then we removed all vectors. We then inspected each of the inoculated plants for evidence of Pierce's disease symptoms. For these inspections, we noted only whether plants showed any of the characteristic leaf scorch symptoms (i.e., symptom presence/absence); we did not attempt to score relative levels of disease severity. Next we collected petiole samples from each of the inoculated plants. These petioles were plate cultured with dilution to estimate $X$. fastidiosa infection level in inoculated plants at approximately the time vectors were present (Hill and Purcell 1995). Finally, the initially healthy plants remained in the medium temperature room for up to 3 months after all trials were completed. At the end of this period, we noted whether they showed any disease symptoms and plate cultured petiole samples to determine if the initially healthy plants had become infected with $X$. fastidiosa during the trial.

Between the six successive dates we replaced plants with a new pair, such that among dates the cages were independent of each other (i.e., nonrepeated measures). A total of 96 cages were used, with eight replicates of each temperature treatment at each of the six dates. Those inoculated plants that did not test positive at the end of a given trial were plate cultured again at the end of the study to determine whether they were successfully infected. Five cages for which the inoculated plant was not successfully infected with $X$. fastidiosa were excluded from analysis.

Data analysis. It is worth noting that we were limited to a single greenhouse for each of the temperature treatment levels. This logistical constraint introduces the potential for autocorrelation in some the response variables regarding symptomology and infectiousness in the inoculated plants-though measures of vector preference and pathogen spread, which were applied at the cage level, would be arguably more truly independent. Regardless, for the purposes of analysis, we necessarily treated all plants as independent replicates.

We analyzed effects on $X$. fastidiosa incubation rate by comparing the proportion of inoculated vines whose infection level, based on plate culturing, was more than $10^{4} \mathrm{CFU} / \mathrm{g}$ of plant material at the end of the transmission trial. In other words, plant infectivity was treated categorically based on whether it was above the level that is known to be required for efficient acquisition by these vectors (Hill and Purcell 1997). We tested for effects of temperature treatment as a fixed effect and day (i.e., number of days postinoculation) as a covariate using a generalized linear model with binomial error (Crawley 2007). We used stepwise deletion of nonsignificant interactions from the full model, with goodness-of-fit $F$ tests, to determine the minimum adequate model (Crawley 2007).

Effects on Pierce's disease symptom onset were analyzed by comparing the proportion of inoculated vines showing disease symptoms at the end of each trial. As with plant infection, we used a generalized linear model with binomial error to test for effects of temperature as a fixed effect and day as a covariate.

The number of insects on each plant was used as a snapshot estimate of vector preference for plants differing in infection status. In each cage, we calculated the proportion of insects found on the inoculated plant, ignoring the few insects that were on the cage itself. Thus, 0.5 would indicate no vector preference, less than 0.5 would indicate preference for the healthy plant, and greater than 0.5 would indicate preference for pathogen-inoculated plants. This proportion was compared using a two-way analysis of variance (ANOVA), in which temperature was a fixed effect and day was a covariate (Crawley 2007). Although it is not uncommon for proportion data to violate the assumptions of such linear models, requiring transformation or other modeling approaches, we inspected the data for normality and homogeneous variances and 
found no evidence that either assumption was violated. Thus, the ANOVA was run on the raw, untransformed, proportions. We also tested whether the intercepts for high and low temperatures differed significantly from 0.5 with separate $t$ tests.

The final set of analyses relate to the potential for pathogen spread, which we estimated by evaluating whether those initially healthy plants became infected with $X$. fastidiosa during a given trial. We used a generalized linear model with binomial error to test for effects of temperature and day, both as fixed effects. A significant interaction was followed-up with pairwise Fisher's exact tests.

\section{RESULTS}

Epidemiological modeling. Again, analysis of the model concentrated largely on the transient dynamics and the likelihood of disease outbreak (i.e., $R_{0}>1$ ) to more closely relate to the experiment in the second half of the study (i.e., under what conditions is pathogen spread most/least likely). Results from the model showed that the incubation rate of exposed hosts and the rate of symptom onset ( $\gamma$ and $\delta$, respectively) can both strongly affect disease dynamics, but not necessarily in an equivalent manner. More rapid transition from the exposed to asymptomatic-infectious host state (i.e., higher $\gamma$ ) consistently increased incidence of both infectious host categories (Fig. 1). More rapid symptom onset (i.e., higher $\delta$ ) favored a greater relative prevalence of symptomaticinfectious compared with asymptomatic-infectious hosts (Fig. 1), but did not necessarily affect the total prevalence of all infectious hosts (i.e., C + I). The prevalence of infection in vectors was highly sensitive to the combination of $\delta$ and vector preference. Higher values of $\delta$ increased the prevalence of infective vectors if vectors prefer symptomatic hosts, but had no effect if vectors do not exhibit preference based on host infection phenotype, and generally reduced the prevalence of infective vectors if vectors avoid (i.e., $p<1$ ) symptoms (Supplementary Fig. S1).

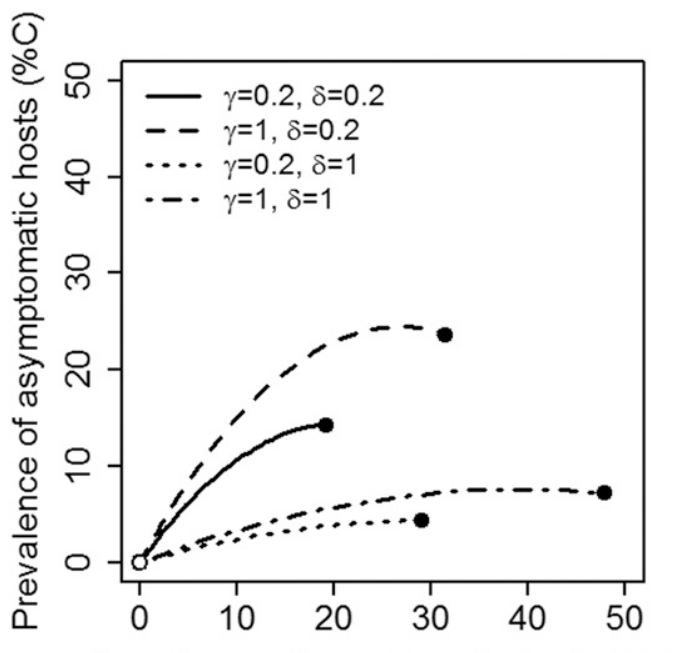

Prevalence of symptomatic hosts (\%l)

Fig. 1. Simulated phase-plane dynamics for the prevalence of symptomatic-infectious hosts versus asymptomatic-infectious hosts for four combinations of incubation rate of exposed hosts $(\gamma)$ and rate of symptom onset $(\delta)$. Prevalence at $t_{0}$ is denoted by the open symbol and final prevalence at $t_{100}$ by the closed symbols. Other parameter values are $\alpha=0.5, \beta=0.4, \mu=0.15, \nu=0.15, p=1, N=100, T=100, S_{0}=100$, $E_{0}=0, I_{0}=0, C_{0}=0, U_{0}=99$, and $V_{0}=0$.
An interaction between vector preference and hypothesized effects of climate is also apparent in the outcomes for $R_{0}$ (Figs. 2 and 3 ). Vector preference for symptoms generally increased the potential for disease outbreak whereas avoidance of symptomatic hosts constrained it (Figs. 2 and 3). More interesting were the divergent effects of accelerated disease phenology based on different assumptions for vector preference. High incubation rates or more rapid symptom onset promoted higher values of $R_{0}$ if vectors
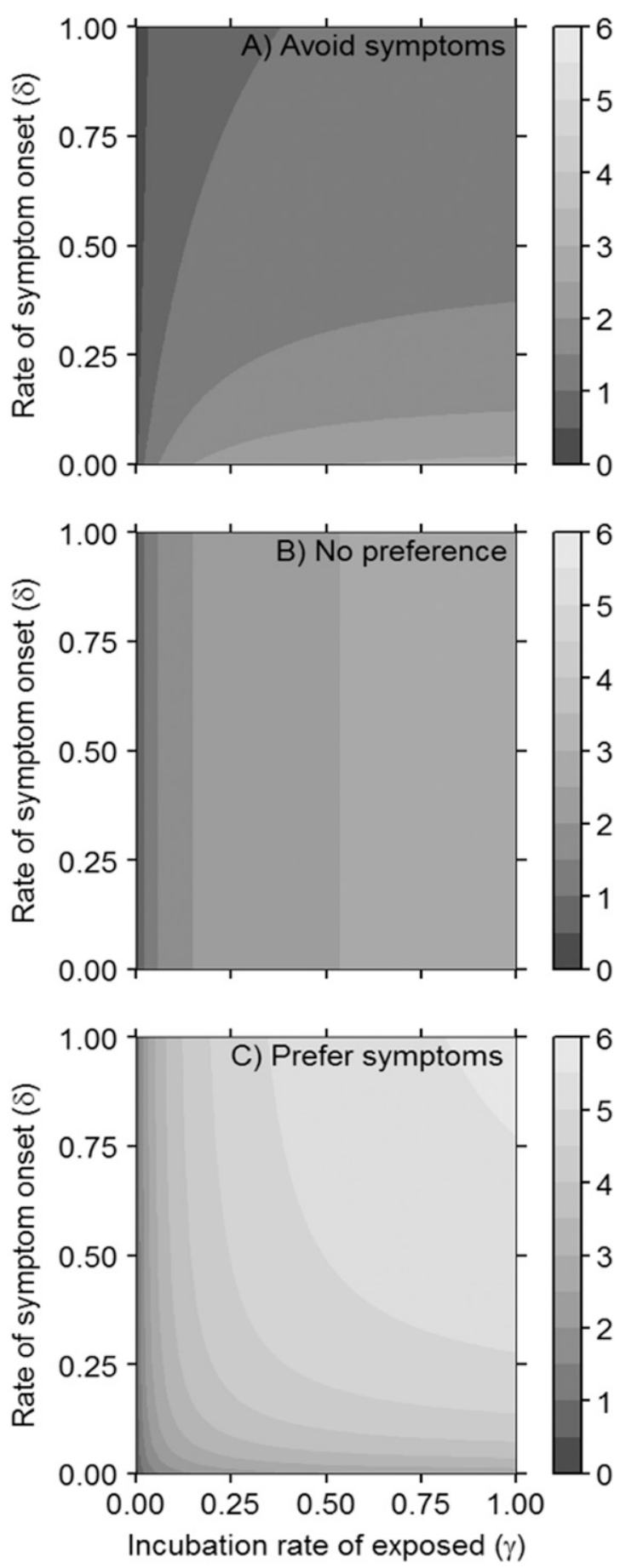

Fig. 2. Pathogen net reproduction rate $\left(R_{0}\right)$ as a function of incubation rate of exposed hosts and rate of symptom onset under scenarios where vectors $\mathbf{A}$, strongly avoid $(p=0.05)$; $\mathbf{B}$, show no preference $(p=1)$; or C, strongly prefer $(p=5)$ symptomatic hosts. Other parameter values are $\alpha=0.4, \beta=0.3, \mu=0.2, \nu=0.2, N=100$, and $T=150$. 
show no preference or prefer symptomatic hosts (Fig. 2B and C). Yet, if vectors avoid symptoms, higher incubation rate favored pathogen outbreak whereas more rapid symptom onset constrained it (Fig. 2A). For pathosystems in which vectors avoid symptoms, the magnitude of avoidance interacts strongly with climate (Fig. 3). A warmer climate favors disease in the absence of vector preference but is also more sensitive to vector avoidance of symptoms. Specifically, for a given incremental reduction in the value of $p, R_{0}$ decreases proportionately more in the warmer conditions compared with cooler conditions, leading to at least as strong constraints on disease outbreaks if vectors strongly avoid symptoms (Fig. 3).

Experiment with $\mathrm{G}$. atropunctata and $\mathrm{X}$. fastidiosa. Over the 15 weeks that plants were in the low and high temperature rooms, mean $( \pm \mathrm{SD})$ day and night temperatures were $22.6 \pm 1.9$ and $17.5 \pm 1.1^{\circ} \mathrm{C}$ and $30.0 \pm 3.1$ and $21.6 \pm 1.4^{\circ} \mathrm{C}$, respectively. Temperatures in the common room in which transmission trials took place were intermediate at $24.7 \pm 2.8$ and $17.9 \pm 1.4^{\circ} \mathrm{C}$ for day and night, respectively.

As noted, 91 of the 96 grapevine cuttings inoculated with $X$. fastidiosa had detectable infections by the end of the study. However, bacterial populations (CFU/g of plant) in these infected plants at the time vectors were exposed to them varied dramatically, from 0 to such high populations that individual colonies could not be counted even after $10^{6}$-fold dilution. The best fit model for the proportion of inoculated plants that were infectious (i.e., $>10^{4}$ CFU/g) included significant effects of temperature $\left(\chi_{1}^{2}=14.563\right.$, $P=0.0001)$ and number of days postinoculation $\left(\chi_{1}^{2}=13.231, P=\right.$ $0.0003)$. Infectivity increased rapidly at the higher temperature with approximately $10 \%$ of plants infectious at the second date, 31 days after inoculation, and up to approximately $80 \%$ infectious at later dates (Fig. 4A). Infectivity in the low temperature plants progressed much more slowly, with the first infections greater than $10^{4} \mathrm{CFU} / \mathrm{g}$ occurring in the fifth trial, 97 days after inoculation.

Symptom expression in the inoculated plants showed patterns that were similar to plant infectivity, although symptoms manifest

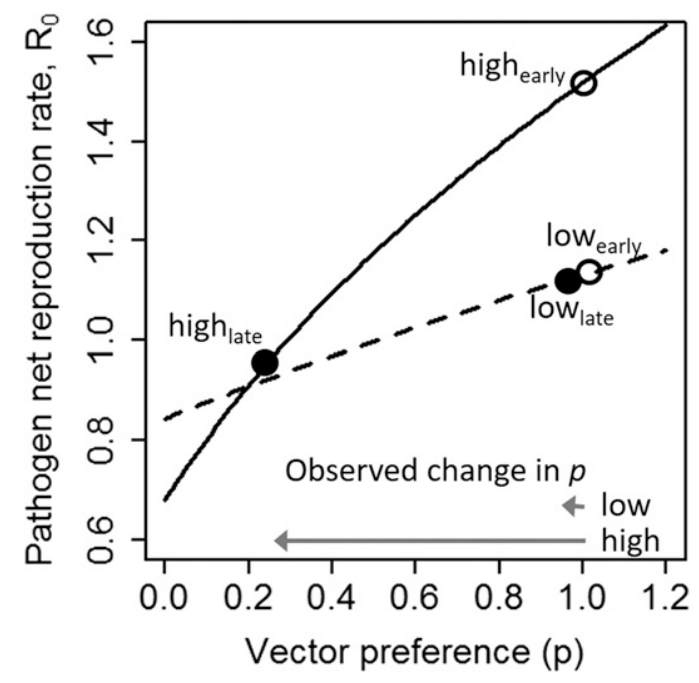

Fig. 3. Model prediction for the effect of vector avoidance of symptoms (i.e., $p<1$ ) on the pathogen net reproduction rate in cool (solid line; $\gamma=$ $0.2, \delta=0.2$ ) versus warm conditions (dashed line; $\gamma=1, \delta=1$ ). Open versus closed symbols reflect approximate changes in vector preference observed in the experiment between early versus late vector introduction dates, which are predicted to lead to a substantially greater reduction in the potential for pathogen spread over time in high temperature versus low temperature conditions. Other parameter values are $\alpha=0.4, \beta=0.3$, $\mu=0.25, v=0.25, N=100$, and $T=150$. slightly more slowly than infections. The best-fit model included significant effects of temperature $\left(\chi_{1}^{2}=14.740, P=0.0001\right)$ and days postinoculation $\left(\chi_{1}^{2}=14.797, P=0.0001\right)$ on the proportion of inoculated plants that showed any disease symptoms. Symptoms manifest quickly in the high temperature plants, with plants showing symptoms by the third trial, 53 days after inoculation (Fig. 4B). The cold temperature plants only started to show Pierce's disease at the last trial, 113 days after inoculation with $X$. fastidiosa.

The proportion of $G$. atropunctata found on the infected plant was used as an indicator of vector preference based on plant infection status. This proportion was not affected significantly by the main effects of temperature $\left(F_{1,86}=1.571, P=0.2135\right)$ or days postinoculation $\left(F_{1,86}=1.221, P=0.2723\right)$, but the interaction was significant $\left(F_{1,86}=5.773, P=0.0184\right)$. The intercepts did not differ significantly from 0.5 for vectors in low $(b \pm \mathrm{SE}=-0.632 \pm$ $\left.0.101 ; t_{1}=-1.311, P=0.197\right)$ or high temperatures $(b \pm$ $\left.\mathrm{SE}=-0.417 \pm 0.091 ; t_{1}=1.030, P=0.3088\right)$. Vectors in both treatments started in the first dates near $50 \%$ on the inoculated plant, indicating no preference (Fig. 5). However, although the proportion of vectors on the inoculated plant did not change appreciably in the low temperature $(m \pm \mathrm{SE}=-0.001 \pm 0.001)$, the proportion declined over time in the high temperature $(m \pm \mathrm{SE}=-0.003 \pm$ $0.001)$. Between the first and last vector introduction date, based on
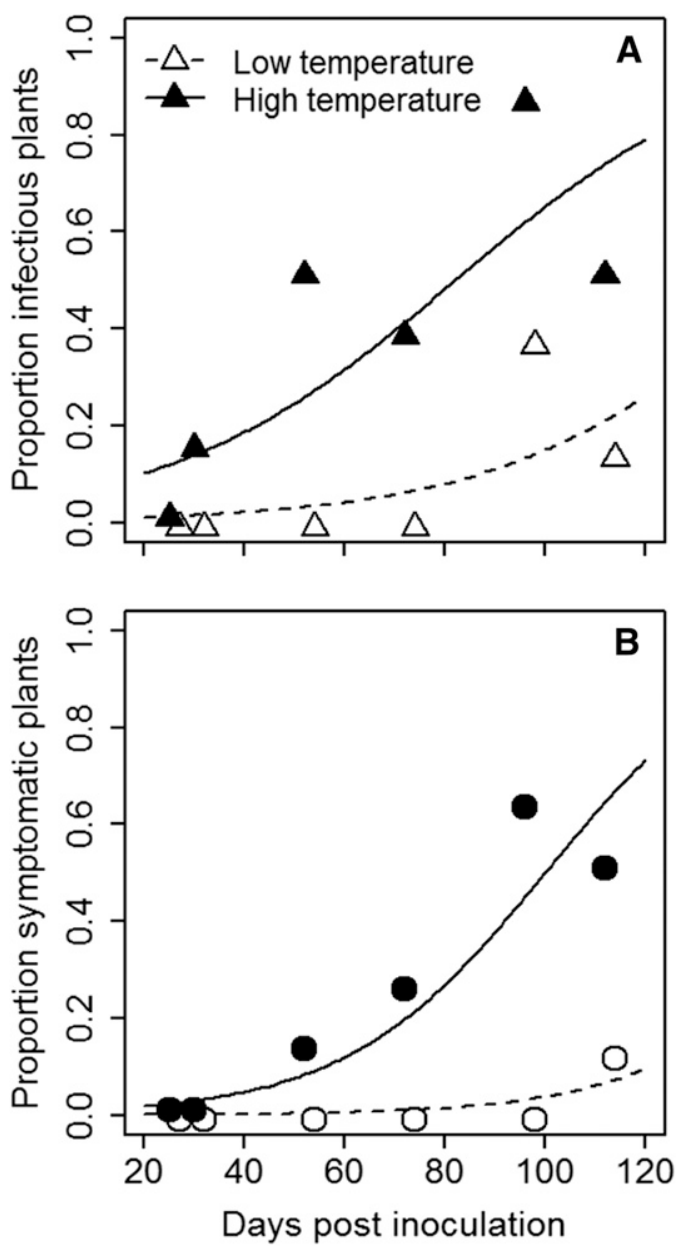

Fig. 4. Proportion of inoculated plants over time that $\mathbf{A}$, have high enough Xylella fastidiosa infection levels to be adequate pathogen sources (i.e., $>10^{4} \mathrm{CFU} / \mathrm{g}$ of plant) or B, are showing Pierce's disease symptoms. Open and closed symbols denote the overall proportions for plants raised at low temperature and at higher temperature, respectively. Lines denote fit of the generalized linear model. Points are offset slightly for clarity. 
the fit of the linear model, observed numbers of vectors on the infected plants equated to $p$ changing from 1 (i.e., $1: 1,50 \%$ on infected; no preference) to less than 0.25 (i.e., 1:4, $20 \%$ on infected; avoidance) in the high temperature and did not change significantly from 1 in the cold temperature. According to the modeling, the disproportionately greater increase in vector avoidance of symptoms observed over time in the high but not the low temperatures may constrain substantially pathogen spread over time (note overlaid points in Figure 3), a prediction that is supported qualitatively by the empirical estimates of spread.

The proportion of initially healthy plants that became infected with $X$. fastidiosa was used as a metric of pathogen spread. Overall, vectors spread the pathogen from the inoculated to the initially healthy plant in approximately $16 \%$ of replicates (14 of 91 cages), with spread occurring in all but the initial trial. Main effects of temperature $\left(\chi_{1}^{2}=0.419, P=0.5173\right)$ and days postinoculation $\left(\chi_{5}^{2}=9.581, P=0.088\right)$ were not significant, but the interaction was significant $\left(\chi_{5}^{2}=11.438, P=0.0434\right)$. If the first census (in which the vast majority of inoculated plants were not yet infectious) is ignored, the significance of the interaction is more pronounced $\left(\chi_{4}^{2}=11.438, P=0.0221\right)$. In the low temperature treatment, the first cases of pathogen spread occurred in the fourth trial, 73 days postinoculation, with approximately $25 \%$ of replicates showing spread at that date and afterward (Fig. 6). Pathogen spread occurred much earlier in the high temperature treatment, at 31 days postinoculation. However, after an early peak of $60 \%$ of cages showing spread in the high temperature, there was a strong decline over time to zero in the final date.

\section{DISCUSSION}

Mitigating the effect of climate change on disease dynamics requires knowledge of the manner in which climate affects different aspects of a pathosystem. We used a combination of epidemiological modeling and an empirical test to investigate links among temperature, infection dynamics, symptom onset, and pathogen spread in a system where prior research suggests both an important role for climate (Daugherty et al. 2009; Feil and Purcell 2001; Lieth et al. 2012; Son et al. 2009) and strong response by vectors to host symptomology (Daugherty et al. 2011; Marucci et al. 2005). The results indicate that warmer conditions can facilitate pathogen

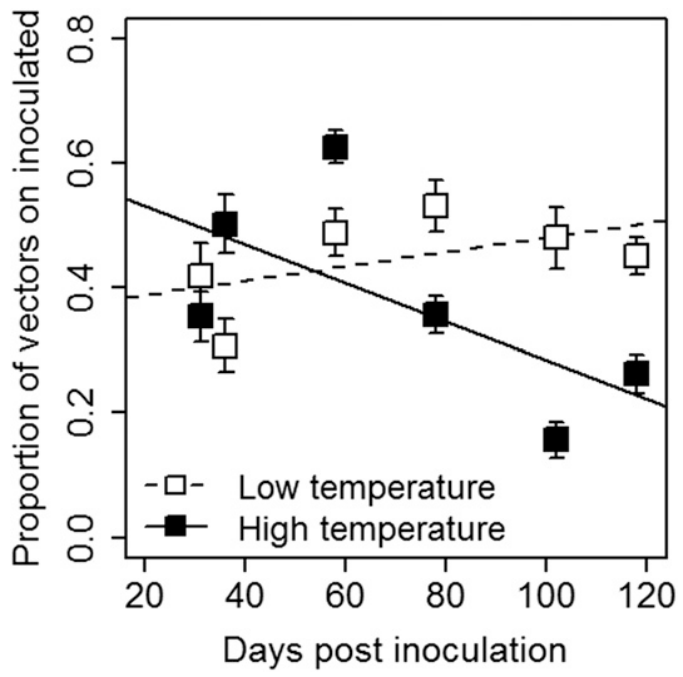

Fig. 5. Mean proportion ( \pm SE) of Graphocephala atropunctata found on the inoculated plant over time. Means of each treatment are shown for clarity. spread, but with effects that are contingent on vector response to disease symptoms.

In the experiment, warmer conditions accentuated the development of high $X$. fastidiosa populations within infected hosts, which is consistent with previous studies of $X$. fastidiosa that showed a generally positive effect of temperature on pathogen multiplication over the same range of temperatures (Feil and Purcell 2001). Other pathosystems have documented relationships between temperature and pathogen incubation rate, though the effect need not be positive and is frequently not monotonic. For example, huanglongbing prevalence in citrus trees varies regionally in part because one of the pathogens associated with the disease is heat sensitive (Lopes et al. 2009). Further studies are needed of intrinsic incubation rate over climate gradients, and corresponding effects on pathogen acquisition, especially given that more rapid incubation should favor higher disease incidence (Zeilinger and Daugherty 2014). Therefore, it is notable that in the current study we found only a tendency for higher likelihood of pathogen spread at higher incubation temperatures.

In addition to increasing incubation rate, warmer conditions favored more rapid onset of disease symptoms. Similar effects of temperature have been noted for the onset or severity of directly transmitted plant pathogens (Lovell et al. 2004). Although we did not quantify symptom severity, anecdotally, infected hosts showed more severe disease symptoms at later dates, particularly at the higher temperature. Regardless, disease symptom onset was not coincident with the onset of host infectiousness. Intrinsic incubation period and host latency are often used synonymously in the description of some pathogens where they coincide temporally or where symptom severity is used to define infectivity (e.g., number of lesions). Yet, infectiousness can often predate symptomology. For example, in huanglongbing, pathogen acquisition by vectors may predate the first disease symptoms by several months or more (Coletta-Filho et al. 2014). A substantially delayed symptom onset compared with incubation period may undercut the effective application of interventions, such as roguing of infected hosts, if relying solely on symptom expression (ColettaFilho et al. 2014). Additionally, the relative duration of incubation periods and asymptomatic phases may be an important

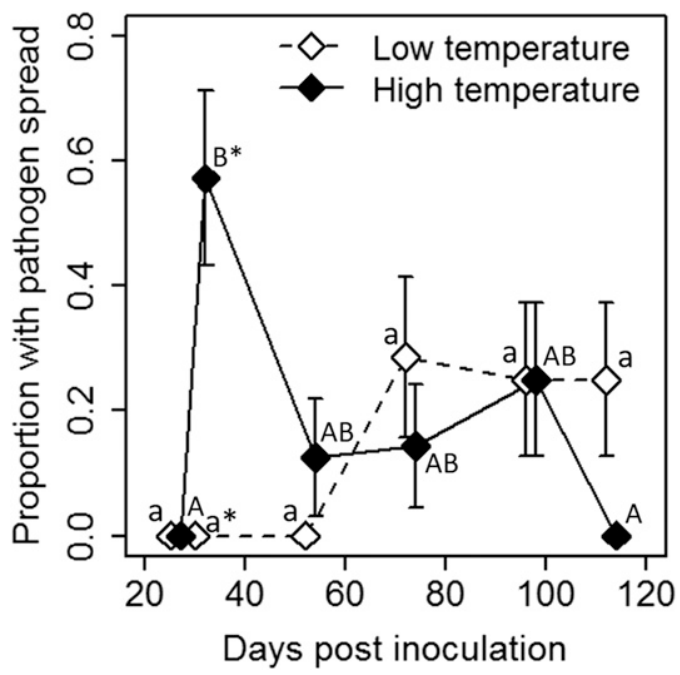

Fig. 6. Proportion of cages over time in which infection spread to the initially healthy plant. Different lowercase and uppercase letters represent significant or marginally significant difference among time points at the low and high temperatures, respectively. ${ }^{*}$ denotes days for which there was a significant difference between the temperatures. 
determinant of disease dynamics when vectors respond strongly to host disease status.

Vectors of animal (Cornet et al. 2013; O'Shea et al. 2002) and plant (Eigenbrode et al. 2002; Ingwell et al. 2012; Mauck et al. 2010) pathogens may respond broadly to changes in host phenotype following infection. In the current study, we quantified vector preference in an admittedly simplistic manner by counting the number of vectors on each host at the end of a trial. This approach was taken to minimize disturbing the vectors in a way that might encourage artifactual pathogen spread. A prior field experiment (Daugherty et al. 2011) suggested that, although there appears to be some individual variation, sharpshooters typically do not move very often, particularly after moving onto healthy grapevines. Thus, our snapshot measure should provide a reasonable proxy for vector preference. We observed that vectors were progressively less likely to be found on inoculated hosts that had been infected longer, at least in the warmer conditions. Vectors showed no preference for infection status for hosts raised in the cooler conditions, which showed very delayed symptom onset. These results are not only congruent with prior studies of vector behavior (Daugherty et al. 2011; Marucci et al. 2005), but also a more recent study of vector feeding based on citrus variegated chlorosis infection status (De Miranda et al. 2013). In the latter study, vectors were less likely to feed on symptomatic hosts and engaged in truncated feeding bouts on symptomatic hosts, whose vascular function is compromised, relative to asymptomatic-infected hosts. Thus, disease symptom onset can trigger a strong response by vectors in a manner that may influence pathogen acquisition.

Our analysis expanded on previous theory (McElhany et al. 1995; Sisterson 2008; Zeilinger and Daugherty 2014) by investigating the extent to which vector behavior might interact with presumed climate-mediated changes in host incubation rate and symptom onset. The results suggest that if vectors either show no preference or prefer symptomatic hosts, warmer conditions should consistently favor a greater potential for disease outbreaks. Yet, if a vector avoids symptomatic hosts, disease dynamics will depend strongly on the nature with which climate affects the pathosystem. For such vectors, conditions that accelerate the incubation rate but not symptom onset maximize disease incidence whereas accelerated symptom onset but not incubation rate minimize it. In other words, vector avoidance of symptoms may constrain the extent to which climate can facilitate disease outbreaks, by limiting the role symptomatic hosts play as acquisition sources. This prediction was supported generally by the results of our experiment, for which disease spread was not consistently greater under warmer conditions. Later on, as symptoms manifest in the warmer conditions, vectors increasingly avoided infected hosts, and pathogen spread declined. Collectively these results provide novel evidence that not only may climate alter vector preference for hosts in a dynamic manner (Werner et al. 2009), but that such changes are epidemiologically significant. Importantly, this body of literature and our present work only considers changes in vector preference from warming mediated through changes in host plant quality. Warming could also influence vector feeding behavior directly, for example, through increasing movement rates; the epidemiological consequences of such direct effects on vector feeding deserve further consideration.

The generality of the conclusion that climate and vector behavior interact to determine disease dynamics is likely to depend on key attributes of a given pathosystem, including the amount of time between the onset of host infectiousness and onset of symptoms. As noted, some pathosystems can have protracted asymptomaticinfectious periods (i.e., several months; Coletta-Filho et al. 2014). Yet for others, especially those in which vectors respond to rapidly induced volatile cues from infected hosts (Eigenbrode et al. 2002), this gap is likely to be much shorter (i.e., days to a week). Another important consideration is the nature of vector preference. In many systems, unlike sharpshooters and X. fastidiosa, vectors are attracted to infected hosts (Eigenbrode et al. 2002; Mauck et al. 2010). For such systems, more rapid symptom onset should favor greater disease incidence, with presumably a greater potential for warmer conditions that favor pathogen multiplication to promote, unchecked, disease outbreaks. A better understanding of the interplay among these factors is needed to predict specifically how pathosystems will respond to a changing climate.

\section{ACKNOWLEDGMENTS}

We thank N. Killiny for providing insects, S. Purcell for helpful discussion throughout this project, and K. Anderson for comments on an earlier draft of this manuscript. R. P. P. Almeida was supported by a grant from the Pierce's Disease Control Program. M. P. Daugherty was supported by funds from USDA-CSREES and from the Pierce's Disease Control Program.

\section{LITERATURE CITED}

Blua, M. J., and Perring, T. M. 1992. Effects of zucchini yellow mosaic virus on colonization and feeding behavior of Aphis gossypii (Homoptera: Aphididae) alatae. Environ. Entomol. 21:578-585.

Canto, T., Aranda, M. A., and Fereres, A. 2009. Climate change effects on physiology and population processes of hosts and vectors that influence the spread of hemipteran-borne plant viruses. Glob. Change Biol. 15:1884-1894.

Carpenter, S., Wilson, A., Barber, J., Veronesi, E., Mellor, P., Venter, G., and Gubbins, S. 2011. Temperature dependence of the extrinsic incubation period of orbiviruses in Culicoides biting midges. PLoS One 6:e27987.

Coletta-Filho, H. D., Daugherty, M. P., Ferreira, C., and Lopes, J. R. S. 2014. Temporal progression of 'Candidatus Liberibacter asiaticus' infection in citrus and acquisition efficiency by Diaphorina citri. Phytopathology 104: 416-421.

Cornet, S., Nicot, A., Rivero, A., and Gandon, S. 2013. Malaria infection increases bird attractiveness to uninfected mosquitoes. Ecol. Lett. 16: 323-329.

Crawley, M. J. 2007. The R Book. John Wiley \& Sons, England. doi:10.1002/ 9780470515075

Daugherty, M. P., and Almeida, R. P. P. 2009. Quantifying the transmission process of a plant pathogen and its vectors: Xylella fastidiosa and sharpshooters in grapevines. Entomol. Exp. Appl. 132:84-92.

Daugherty, M. P., Bosco, D., and Almeida, R. P. P. 2009. Temperature mediates vector transmission efficiency: Inoculum supply and plant infection dynamics. Ann. Appl. Biol. 155:361-369.

Daugherty, M. P., Lopes, J. R. S., and Almeida, R. P. P. 2010. Vector within-host feeding preference mediates transmission of a heterogeneously distributed pathogen. Ecol. Entomol. 35:360-366.

Daugherty, M. P., Rashed, A., Almeida, R. P. P., and Perring, T. M. 2011. Vector preference for host infection status: Sharpshooter movement and Xylella fastidiosa transmission. Ecol. Entomol. 36:654-662.

De Miranda, M. P., Villada, E. S., Lopes, S. A., Fereres, A., and Lopes, J. R. S. 2013. Influence of citrus plants infected with Xylella fastidiosa on stylet penetration activities of Bucephalogonia xanthophis (Hemiptera: Cicadellidae). Ann. Entomol. Soc. Am. 106:610-618.

Eigenbrode, S. D., Ding, H., Shiel, P., and Berger, P. H. 2002. Volatiles from potato plants infected with potato leafroll virus attract and arrest the virus vector, Myzus persicae (Homoptera: Aphididae). Proc. R. Soc. Lond. B 269: 455-460.

Feil, H., and Purcell, A. H. 2001. Temperature-dependent growth and survival of Xylella fastidiosa in vitro and in potted grapevines. Plant Dis. 85:1230-1234.

Gruber, B. R., and Daugherty, M. P. 2013. Predicting the effects of seasonality on the risk of pathogen spread in vineyards: Vector pressure, natural infectivity, and host recovery. Plant Pathol. 62:194-204.

Hill, B. L., and Purcell, A. H. 1995. Acquisition and retention of Xylella fastidiosa by an efficient vector, Graphocephala atropunctata. Phytopathology 85:209-212. 
Hill, B. L., and Purcell, A. H. 1997. Populations of Xylella fastidiosa in plants required for transmission by an efficient vector. Phytopathology 87:1197-1201.

Ingwell, L. L., Eigenbrode, S. D., and Bosque-Pérez, N. A. 2012. Plant viruses alter insect behavior to enhance their spread. Sci. Rep. 2:578.

Lafferty, K. D. 2009. The ecology of climate change and infectious disease. Ecology 90:888-900.

Lieth, J. H., Meyer, M. M., Yeo, K.-H., and Kirkpatrick, B. C. 2012. Modeling cold curing of Pierce's disease in Vitis vinifera 'Pinot Noir' and 'Cabernet Sauvignon' grapevines in California. Phytopathology 101:1492-1500.

Lopes, S. A., Frare, G. F., Bertolini, E., Cambra, M., Fernandes, N. G., Ayres, A. J., Marin, D. R., and Bové, J. M. 2009. Liberibacters associated with citrus Huanglongbing in Brazil: 'Candidatus Liberibacter asiaticus' is heat tolerant, 'Ca. L. americanus' is heat sensitive. Plant Dis. 93:257-262.

Lovell, D. J., Hunter, T., Powers, S. J., Parker, S. R., and Van den Bosch, F. 2004. Effect of temperature on latent period of Septoria leaf blotch on winter wheat under outdoor conditions. Plant Pathol. 53:170-181.

Marucci, R. C., Lopes, J. R. S., Vendramim, J. D., and Corrente, J. E. 2005. Influence of Xylella fastidiosa infection of citrus on host selection by leafhopper vectors. Entomol. Exp. Appl. 117:95-103.

Mauck, K. E., De Moraes, C. M., and Mescher, M. C. 2010. Deceptive chemical signals induced by a plant virus attract insect vectors to inferior hosts. Proc. Natl. Acad. Sci. USA 107:3600-3605.

McElhany, P., Real, L. A., and Power, A. G. 1995. Vector preference and disease dynamics: A study of Barley yellow dwarf virus. Ecology 76:444-457.

Munyaneza, J. E., Sengoda, V. G., Buchman, J. L., and Fisher, T. W. 2012. Effects of temperature on 'Candidatus Liberibacter solanacearum' and zebra chip potato disease symptom development. Plant Dis. 96:18-23.

O'Shea, B., Rebollar-Tellez, E., Ward, R. D., Hamilton, J. G. C., El Naiem, D., and Polwart, A. 2002. Enhanced sandfly attraction to Leishmania-infected hosts. Trans. R. Soc. Trop. Med. Hyg. 96:117-118.

Purcell, A. H. 1975. Role of the blue-green sharpshooter, Hordnia circellata, in the epidemiology of Pierce's disease of grapevines. Environ. Entomol. 4:745-752.
Purcell, A. H. 1981. Vector preference and inoculation efficiency as components of resistance to Pierce's disease in European grape cultivars. Phytopathology 71:429-435.

Purcell, A. H. 1997. Xylella fastidiosa, a regional problem or a global threat? J. Plant Pathol. 79:99-105.

Richards, S. L., Mores, C. N., Lord, C. C., and Tabachnick, W. J. 2007. Impact of extrinsic incubation temperature on virus exposure on vector competence of Culex pipiens quinquefasciatus Say (Diptera: Culicidae) for West Nile virus. Vector Borne and Zoonotics Dis. 7:629-636.

Rohr, J. R., Dobson, A. P., Johnson, P. T. J., Kilpatrick, A. M., Paull, S. H., Raffel, T. R., Ruiz-Moreno, D., and Thomas, M. B. 2011. Frontiers in climate change-disease research. Trends Ecol. Evol. 26:270-277.

Savage, L. T., Reich, R. M., Hartley, L. M., Stapp, P., and Antolin, M. F. 2011. Climate, soils, and connectivity predict plague epizootics in black-tailed prairie dogs (Cynomys ludovicianus). Ecol. Appl. 21:2933-2943.

Sisterson, M. S. 2008. Effects of insect-vector preference for healthy or infected plants on pathogen spread: insights from a model. J. Econ. Entomol. 101:1-8.

Son, Y., Groves, R. L., Daane, K. M., Morgan, D. J. W., and Johnson, M. W. 2009. Influence of temperature on Homalodisca vitripennis (Hemiptera: Cicadellidae) survival under various feeding conditions. Environ. Entomol. 38:1485-1495.

Son, Y., Groves, R. L., Daane, K. M., Morgan, D. J. W., Krugner, R., and Johnson, M. W. 2010. Estimation of feeding thresholds for Homalodisca vitripennis (Hemiptera: Cicadellidae) and its application to prediction of overwinter mortality. Environ. Entomol. 39:1264-1275.

Werner, B. J., Mowry, T. M., Bosque-Perez, N. A., Ding, H., and Eigenbrode, S. D. 2009. Changes in green peach aphid responses to potato leafroll virus-induced volatiles emitted during disease progression. Environ. Entomol. 38:1429-1438.

Zeilinger, A., and Daugherty, M. P. 2014. Vector preference and host defense against infection interact to determine disease dynamics. Oikos 123:613-622. 